(rs11640851 and rs8052394) and two MT2A SNPs (rs10636 and rs28366003). The MT1A2A SNPs combination was dividedinto 16 groups. Data were treated for descriptive analysis, one-way ANOVA, and multiple linear regressions by SPSS.

Results Urine uric acid had significantly negative association with time-weighted index of cumulative blood lead (TWICL) after adjusting other potential confounders except 16 groups of MT1A2A SNPs combination. Moreover, the association was modified by 16 groups of SNPs combination. For urine uric acid, group 6th and group 12th were more susceptible to lead toxicity, while group 5th was lesssusceptible to lead toxicity. On the other hand, urinary NAG was positively associated with TWICL no matter whether the 16 groups were adjusted or not.

Conclusion Urinary uric acid and urinary NAG might be considered as proper indicators of lead nephrotoxicity. Moreover, MT1A2A combination 16 groups were modifiers in the relationship between urinary uric acid and TWICL. The group 5 th was positive association while group 6th and 12th were negative association with TWICL on urinary uric acid

\section{BONE EFFECT UNDER CAUSED BY CO-EXPOSURE TO FLUORIDE AND ARSENIC}

1,2 Feng Hong*, 1,2Qi-bing Zeng* ${ }^{*}{ }^{1,2}$ Chong Zheng ${ }^{*},{ }^{1,2}$ Xiao-tian Song* ${ }^{*}$ 'Department of Toxicology, School of Public Health, Guizhou Medical University, Guiyang, Guizhou, China; ${ }^{2}$ Key Laboratory of Environmental Pollution Monitoring and Disease Control, Ministry of Education, China

\subsection{6/oemed-2018-ICOHabstracts. 1438}

Introduction Chronic exposure to combined fluoride and arsenic continue to be a major public health problem worldwide. Although there have been reports in the literature about the toxicity of arsenic and fluoride individually, there is very little known about the combined effects of these two elements. In this study, based on the population and in vivo, the aim is to explore the combined bone toxicity of fluorine and arsenic and provides a scientific basis for the mechanism, prevention and control of endemic combined fluorine-arsenic poisoning on bone toxicity.

Methods The population-based study group was selected from people living in fluoride-arsenic polluted areas due to burning coal in China. The total number of participants was 196 to observing the changes in gene and protein expression of PTH/ PKA/AP1 signalling pathway. A total of 90 weaned specific pathogen-free SD rats were randomly assigned to a group to explore the role of PTH-cAMP-PKA signalling pathway on bone toxicity of rats exposed to fluoride and arsenic.

Results Fluoride can increase the expression levels of PTH, PKA, and AP1, but arsenic can only affect the expression of AP1; fluoride and arsenic have an interaction on the expression of AP1, c-fos and Runx2. Fluoride exposure can affect the metabolism of collagen and bone resorption, and arsenic exposure main affect bone resorption, fluoride and arsenic coexposure have a more significant effect on bone resorption.

Conclusion PTH-PKA-AP1 and PTH-cAMP-PKA signalling pathway may play an important role in bone toxicity of fluoride. Arsenic can affect the expression of c-fos and Runx2, thereby affecting the expression of transcription factor AP1, MMP-9, RANKL and Osterix, indirectly involved in fluoride induced bone toxicity. The main sign of bone damage under the exposure level of the study is osteosclerosis and main combined bone toxicity of fluoride and arsenic showed antagonistic effects

\section{LOW LEVEL URINE ARSENIC CONCENTRATION AND OBSTRUCTIVE PULMONARY DISEASE AMONG U.S. WORKERS}

1,2ED Amster*, 'David Christiani. 'University of Haifa, School of Public Health, Department of Occupational Health, Israel; ${ }^{2}$ Harvard University, T.H. Chan School of Public Health, USA

10.1136/oemed-2018-ICOHabstracts. 1439

Introduction Arsenic (As) is a known carcinogen commonly found in drinking water. An emerging body of evidence suggests that exposure to inorganic arsenic may be associated with non-malignant respiratory disease. The aim of this study was to determine whether there is an association between As exposure at levels seen among workers in the United States and prevalence of asthma, emphysema, chronic bronchitis, and respiratory symptoms.

Methods Urinary As was collected from 5365 participants from the combined 2003-2006 National Health and Nutrition Examination Survey (NHANES) cohorts. Two methods to adjust for organic As component were incorporated into the statistical model. Linear and logistic regression models compared urinary As adjusted for organic As with diagnoses of obstructive pulmonary disease and respiratory symptoms.

Results Geometric mean concentration of urinary As were not significantly different between participants with and those without asthma, chronic bronchitis, and emphysema. Odds of having asthma was 0.71 for participants with the highest quintile of urinary As $(\geq 17.23 \mu \mathrm{g} / \mathrm{dl})$ when compared to the lowest quintile $(\leq 3.52 \mu \mathrm{g} / \mathrm{dl})$. A significant association was found between increasing urinary As concentration and decreasing age, male gender, and non-'white' race.

Conclusion A significant association between urinary As and obstructive pulmonary disease and symptoms was not demonstrated in the U.S. working population

\section{BONE METABOLISM ABNORMALITY AND RENAL DYSFUNCTION IN CADMIUM EXPOSED FARMER FROM THAILAND}

${ }^{1} \mathrm{~K}$ Nambunmee*, ${ }^{2} \mathrm{M}$ Nishijo, ${ }^{3} \mathrm{~W}$ Swaddiwudhipong, ${ }^{4} \mathrm{~W}$ Ruangyuttikarn. ${ }^{1}$ Occupational Health and Safety, Major of Public Health, School of Health Science, Mae Fah Luang University, Thailand; '2Department of Public Health, Kanazawa Medical University, Ishikawa, Japan; ${ }^{3}$ Department of Community and Social Medicine, Mae Sot General Hospital, Tak Province, Thailand; ${ }^{4}$ Department of Forensic Medicine, Faculty of Medicine, Chiang Mai University, Thailand

\subsection{6/oemed-2018-ICOHabstracts. 1440}

Introduction Paddy fields in Mae Sot, Tak province of Thailand are polluted with cadmium (Cd) over safe level. Farmers exposed $\mathrm{Cd}$ from contaminated rice consumption and the high prevalence of kidney dysfunction was shown in the exposed population. Bone fracture is another Cd related pathology which shown in elderly with high exposure level. The identification of high risk individuals will be advantageous to provide suitable health promotion and to prevent severe pathology.This study was done to compare bone fracture risk between glomerular dysfunction, proximal tubular dysfunction and Calcium $(\mathrm{Ca})$ handling abnormality.

Methods Serum osteocalcin and cross-linked N-telopeptide of type I collagen to detect bone metabolism abnormalities, whereas glomerular filtration rate, serum cystatin $C$, urinary $\beta 2$-microglobulin $\left(\beta_{2}-\mathrm{MG}\right)$ and fractional excretion of calcium 